\title{
A Tutorial on Fingerprint Recognition ${ }^{1}$
}

\author{
Davide Maltoni \\ Biometric Systems Laboratory - DEIS - University of Bologna \\ via Sacchi 3, 47023, Cesena (FC) - Italy \\ maltoni@csr.unibo.it \\ http://bias.csr.unibo.it/research/biolab
}

\begin{abstract}
This tutorial introduces fingerprint recognition systems and their main components: sensing, feature extraction and matching. The basic technologies are surveyed and some state-of-the-art algorithms are discussed. Due to the extent of this topic it is not possible to provide here all the details and to cover a number of interesting issues such as classification, indexing and multimodal systems. Interested readers can find in [21] a complete and comprehensive guide to fingerprint recognition.
\end{abstract}

\section{Introduction}

A fingerprint-based biometric system is essentially a pattern recognition system that recognizes a person by determining the authenticity of her fingerprint. Depending on the application context, a fingerprint-based biometric system may be called either a verification system or an identification system:

- a verification system authenticates a person's identity by comparing the captured fingerprints with her own biometric template(s) pre-stored in the system. It conducts one-to-one comparison to determine whether the identity claimed by the individual is true;

- an identification system recognizes an individual by searching the entire template database for a match. It conducts one-to-many comparisons to establish the identity of the individual.

Throughout this paper the generic term recognition is used where it is not necessary distinguishing between verification and identification.

The block diagrams of a fingerprint-based verification system and an identification system are depicted in Figure 1; user enrollment, which is common to both tasks is also graphically illustrated. The enrollment module is responsible for registering individuals in the biometric system database (system DB). During the enrollment phase, the fingerprint of an individual is acquired by a fingerprint scanner to produce a raw digital representation. A quality check is generally performed to ensure that the acquired sample can be reliably processed by successive stages. In order to facilitate matching, the raw digital representation is usually further processed by a feature ex-

${ }^{1}$ Portions reprinted from: D. Maltoni, D. Maio, A.K. Jain and S. Prabhakar, "Handbook of Fingerprint Recognition," Springer, 2003. (C2003 Springer.

M. Tistarelli, J. Bigun, and E. Grosso (Eds.): Biometrics School 2003, LNCS 3161, pp. 43-68, 2005.

(C) Springer-Verlag Berlin Heidelberg 2005 
tractor to generate a compact but expressive representation, called a template. The verification task is responsible for verifying individuals at the point of access. During the operation phase, the user's name or PIN (Personal Identification Number) is entered through a keyboard (or a keypad); the biometric reader captures the fingerprint of the individual to be recognized and converts it to a digital format, which is further processed by the feature extractor to produce a compact digital representation. The resulting representation is fed to the feature matcher, which compares it against the template of a single user (retrieved from the system DB based on the user's PIN).
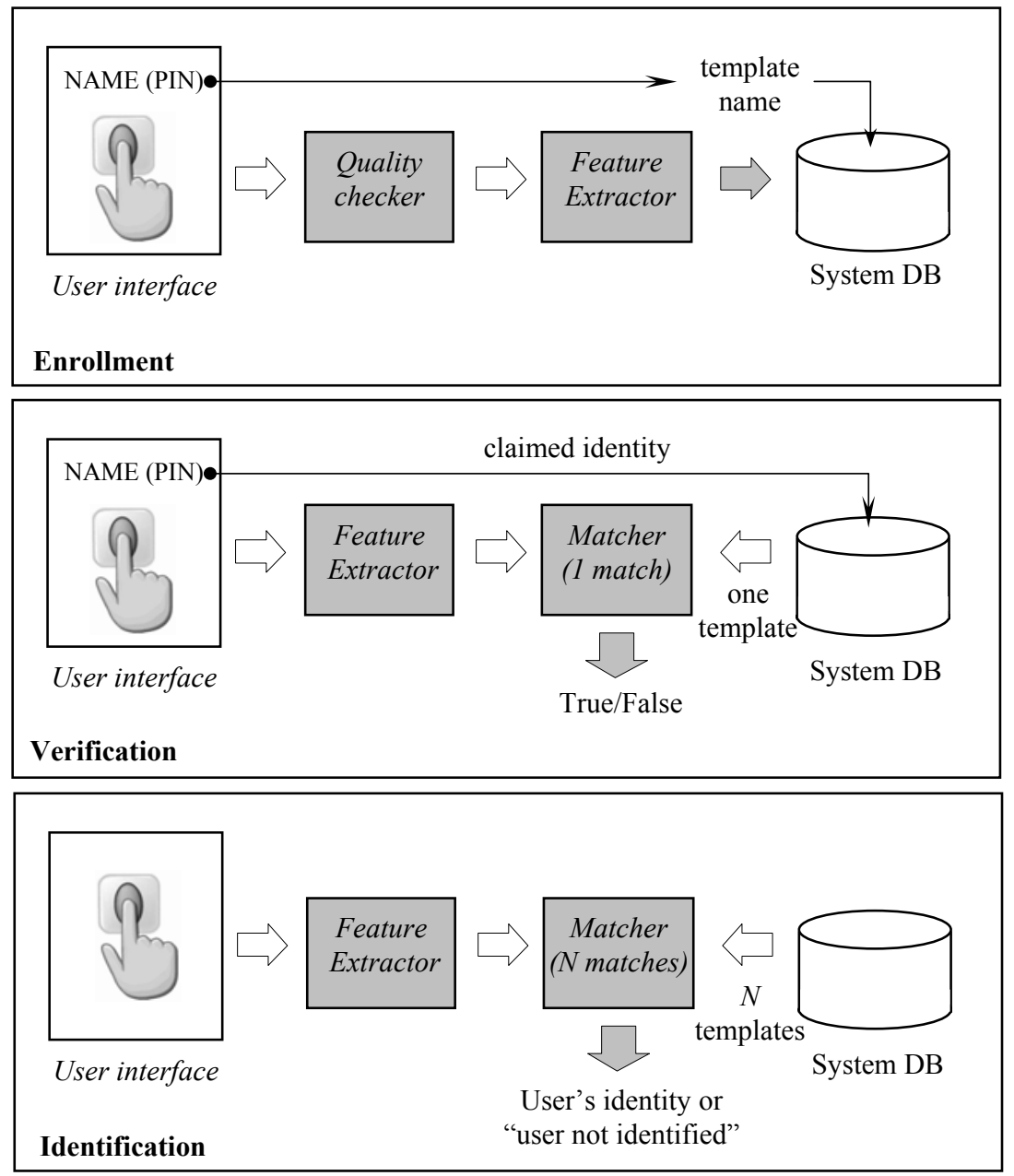

Fig. 1. Block diagrams of enrollment, verification, and identification tasks.

In the identification task, no PIN is provided and the system compares the representation of the input biometric against the templates of all the users in the system database; the output is either the identity of an enrolled user or an alert message such as "user not identified." Because identification in large databases is computationally 
expensive, classification and indexing techniques are often deployed to limit the number of templates that have to be matched against the input.

It is evident from Figure 1 that the main building blocks of any fingerprint-based verification and identification system are: 1) sensing, 2) feature extraction, and 3) matching. The rest of this paper, after a brief subsection introducing biometric system errors, dedicates a separate section to each of the three above topics.

\subsection{Performance of a Fingerprint-Based Recognition System}

No biometric system is perfect. Although the accuracy of fingerprint-based biometric systems can be very high (see FVC2002 results [18]), the output is affected by two types of errors: mistaking biometric measurements from two different fingers to be from the same finger (called false match) and mistaking two biometric measurements from the same finger to be from two different fingers (called false non-match). Note that these two types of errors are also often denoted as false acceptance and false rejection, but the notation "false match/false non-match" is generally preferable because it is not application dependent [21]. There is a strict tradeoff between FMR (false match rate) and FNMR (false non-match rate) in every biometric system [8]. In fact, both FMR and FNMR are functions of a system accuracy threshold $t$. If $t$ is decreased to make the system more tolerant with respect to input variations and noise, then FMR increases; vice versa, if $t$ is raised to make the system more secure, then FNMR increases accordingly. Besides FMR and FNMR, a "compact" value is generally used to summarize the accuracy of a verification system: the Equal-Error Rate (EER) denotes the error rate at the threshold $t$ for which false match rate and false non-match rate are identical: $F M R=F N M R$.

\section{Fingerprint Sensing}

Historically, in law enforcement applications, the acquisition of fingerprint images was performed by using the so-called "ink-technique": the subject's finger was spread with black ink and pressed against a paper card; the card was then scanned by using a common paper-scanner, producing the final digital image. This kind of process is referred to as off-line fingerprint acquisition or off-line sensing. A particular case of off-line sensing is the acquisition of a latent fingerprint from a crime scene. Nowadays, most civil and criminal AFIS accept live-scan digital images acquired by directly sensing the finger surface with an electronic fingerprint scanner. No ink is required in this method, and all that a subject has to do is press his finger against the flat surface of a live-scan scanner. To maximize compatibility between digital fingerprint images and ensure good quality of the acquired fingerprint impressions, the US Criminal Justice Information Services released a set of specifications that regulate the quality and format of both fingerprint images and FBI-compliant off-line/live-scan scanners (ref. to Appendix F and Appendix G of CJIS [6]). 


\subsection{Fingerprint Images}

The main parameters characterizing a digital fingerprint image are as follows.

- Resolution: This indicates the number of dots or pixels per inch (dpi). 500 dpi is the minimum resolution for FBI-compliant scanners and is met by many commercial devices; 250 to 300 dpi is probably the minimum resolution that allows the extraction algorithms to locate the minutiae in fingerprint patterns.

- Area: The size of the rectangular area sensed by a fingerprint scanner is a fundamental parameter. The larger the area, the more ridges and valleys are captured and the more distinctive the fingerprint becomes. An area greater than or equal to $1 \times 1$ square inches (as required by FBI specifications) permits a full plain fingerprint impression to be acquired. In most of the recent fingerprint scanners aimed at non-AFIS market, area is sacrificed to reduce cost and to have a smaller device size. Small-area scanners do not allow a whole fingerprint to be captured, and the users encounter difficulties in re-presenting the same portion of the finger. This may result in a small overlap between different acquisitions of the same finger, leading to false non-match errors.

- Number of pixels: The number of pixels in a fingerprint image can be simply derived by the resolution and the fingerprint area: a scanner working at $r$ dpi over an area of height $(h) \times$ width $(w) i n c h^{2}$ has $r h \times r w$ pixels.

- Dynamic range (or depth): This denotes the number of bits used to encode the intensity value of each pixel. The FBI standard for pixel bit depth is 8 bits, which yields 256 levels of gray.

- Geometric accuracy: This is usually specified by the maximum geometric distortion introduced by the acquisition device, and expressed as a percentage with respect to $x$ and $y$ directions.

- Image quality: It is not easy to precisely define the quality of a fingerprint image, and it is even more difficult to decouple the fingerprint image quality from the intrinsic finger quality or status. In fact, when the ridge prominence is very low (especially for manual workers and elderly people), when the fingers are too moist or too dry, or when they are incorrectly presented, most of the scanners produce poor quality images (see Figure 2).

\subsection{Off-line Acquisition}

- Although the first fingerprint scanners were introduced more than 30 years ago, nowadays, the ink-technique [17] is still used in law enforcement applications. Live-scan acquisition techniques are now being employed in AFIS. As a result, the databases built by law enforcement agencies over a period of time contain both the fingerprint images acquired by off-line scanners and live-scan scanners and the AFIS matching algorithms are expected to interoperate on these different types of images. 


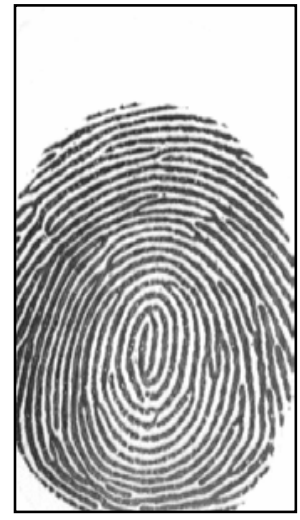

a)

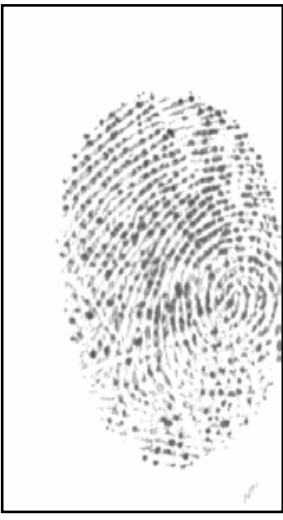

b)

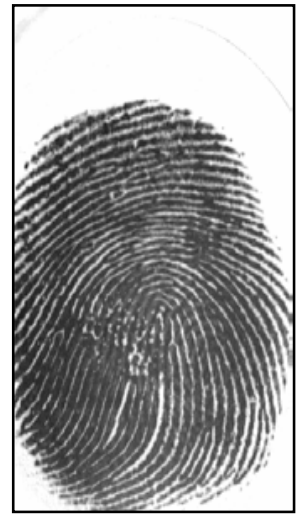

c)

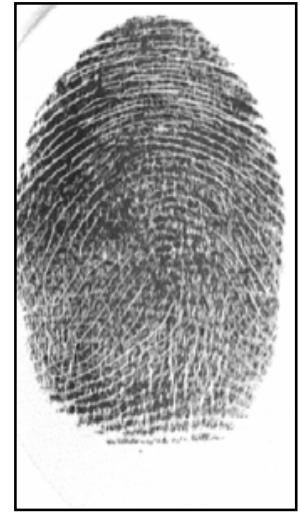

d)

Fig. 2. Examples of fingerprint images acquired with an optical scanner: a) a good quality fingerprint; b) a fingerprint left by a dry finger; c) a fingerprint left by a wet finger, d) an intrinsically bad fingerprint.

In the ink-technique the finger skin is first spread with black ink and then pressed against a paper card; the card is then converted into digital form by means of a paperscanner or by using a high-quality CCD camera (see Figure 3 ). The default resolution is 500 dpi. If not executed with care, the ink-technique produces images including regions with missing information, due to excessive inkiness or due to ink deficiency. On the other hand, an advantage of this technique is the possibility of producing rolled impressions (by rolling "nail-to-nail" a finger against the card, thus producing an unwrapped representation of the whole pattern) which carries more information with respect to the flat (or dab) impressions obtained by simply pressing the finger against the flat surface of a scanner.
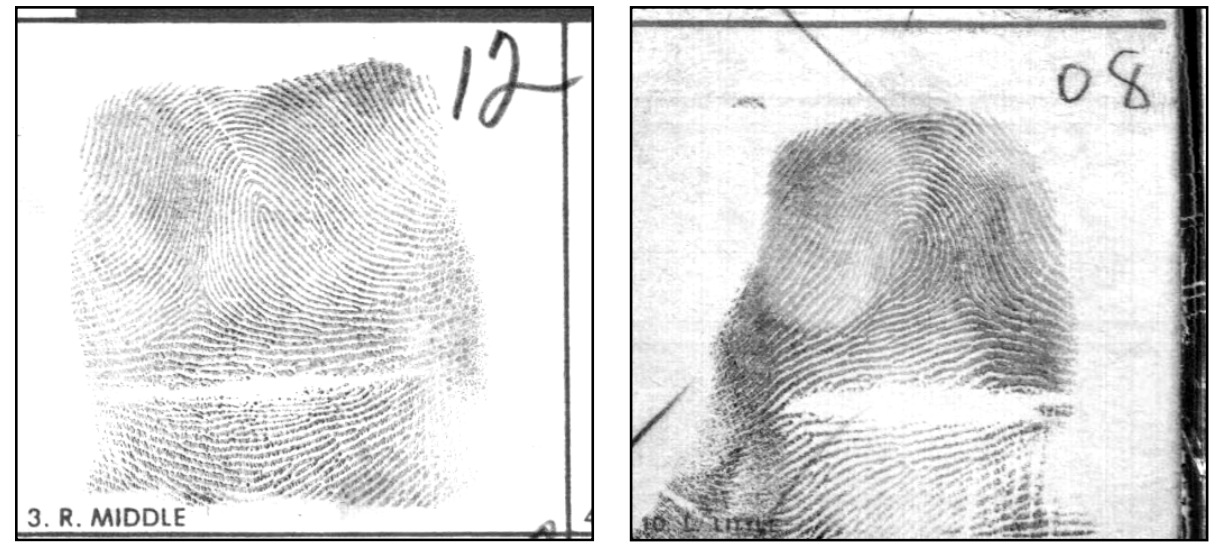

Fig. 3. Rolled fingerprint images acquired off-line with the ink technique. 


\subsection{Live-Scan Sensing}

The most important part of a fingerprint scanner is the sensor (or sensing element), which is the component where the fingerprint image is formed. Almost all the existing sensors belong to one of the three families: optical, solid-state, and ultrasound.

- Optical sensors. Frustrated Total Internal Reflection (FTIR) is the oldest and most used live-scan acquisition technique today. The finger touches the top side of a glass prism, but while the ridges enter in contact with the prism surface, the valleys remain at a certain distance (see Figure 4.a); the left side of the prism is illuminated through a diffused light. The light entering the prism is reflected at the valleys, and absorbed at the ridges. The lack of reflection allows the ridges to be discriminated from the valleys. The light rays exit from the right side of the prism and are focused through a lens onto a CCD or CMOS image sensor. In spite of a generally better image quality and the possibility of larger sensing areas, FTIRbased devices cannot be miniaturized unlike other techniques: optical fibers, electro-optical devices, and solid-state devices.

- Solid-state sensors. Solid-state sensors (also known as silicon sensors) became commercially available in the middle 1990s. All silicon-based sensors consist of an array of pixels, each pixel being a tiny sensor itself. The user directly touches the surface of the silicon: neither optical components nor external CCD/CMOS image sensors are needed. Four main effects have been proposed to convert the physical information into electrical signals: capacitive, thermal, electric field, and piezoelectric. A capacitive sensor is a two-dimensional array of micro-capacitor plates embedded in a chip (see Figure 4.b). The other plate of each microcapacitor is the finger skin itself. Small electrical charges are created between the surface of the finger and each of the silicon plates when a finger is placed on the chip. The magnitude of these electrical charges depends on the distance between the fingerprint surface and the capacitance plates.

- Ultrasound sensors. Ultrasound sensing may be viewed as a kind of echography. Characteristic of sound waves is the ability to penetrate materials, giving a partial echo at each impedance change. An ultrasound sensor is based on sending acoustic signals toward the fingertip and capturing the echo signal (see Figure 4.c). The echo signal is used to compute the range image of the fingerprint and, subsequently, the ridge structure itself. Good quality images may be obtained by this technology. However, the scanner is large with mechanical parts and quite expensive. Moreover, it takes a few seconds to acquire an image. Hence, this technology is not yet mature enough for large-scale production.

Table 1 lists some commercial scanners designed for the non-AFIS markets, whose cost is less than \$200 US. Except for ultrasound scanners, which are not ready for mass-market applications yet, Table 1 includes at least one scanner for each technology. Examples of the same fingerprint (from a good-quality finger, a dry finger, a wet finger, and a poor quality finger, respectively) as acquired by using many of the scanners listed in Table 1 are reported in [21]. 
a)

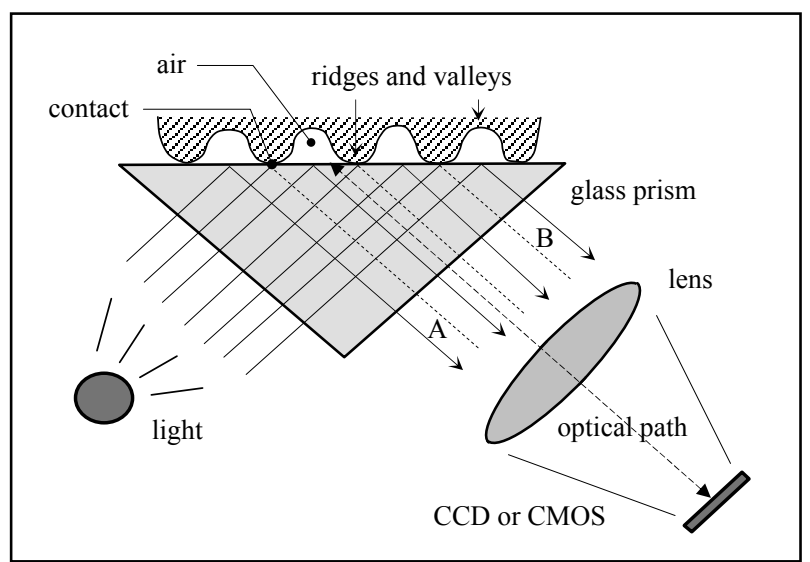

b)

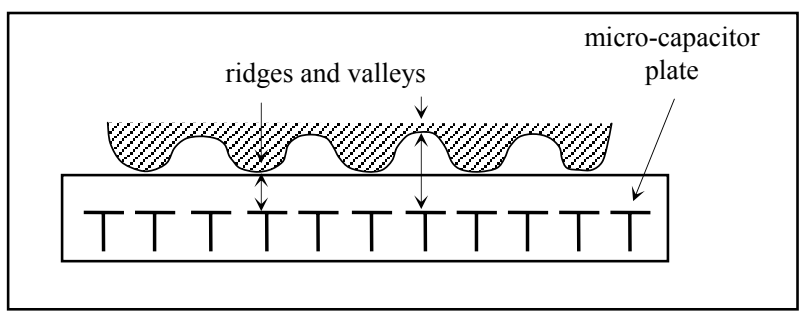

c)

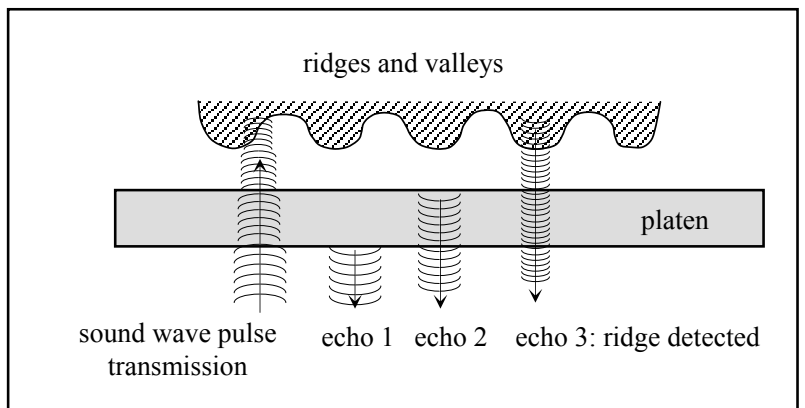

Fig. 4. a) FTIR-based optical fingerprint sensing; b) capacitive sensing; c) the basic principle of the ultrasound technique.

\section{Feature Extraction}

A fingerprint is the reproduction of a fingertip epidermis, produced when a finger is pressed against a smooth surface. The most evident structural characteristic of a fingerprint is a pattern of interleaved ridges and valleys; in a fingerprint image, ridges (also called ridge lines) are dark whereas valleys are bright (see Figure 5.a). Ridges and valleys often run in parallel; sometimes they bifurcate and sometimes they terminate. 
Table 1. Some commercial scanners, grouped by technology. The table reports for each scanner, the resolution, the sensing area, and the number of pixels.

\begin{tabular}{|c|c|c|c|c|c|c|}
\hline & Technology & Company & Model & Dpi & Area $(h \times w)$ & Pixels \\
\hline \multirow{7}{*}{ 苞 } & FTIR & $\begin{array}{c}\text { Biometrika } \\
\text { www.biometrika.it/eng/ }\end{array}$ & FX2000 & 569 & $0.98 " \times 0.52 "$ & $\begin{array}{l}560 \times 296 \\
(165,760) \\
\end{array}$ \\
\hline & FTIR & $\begin{array}{c}\text { Digital Persona } \\
\text { www.digitalpersona.com }\end{array}$ & UareU2000 & 440 & $0.67 " \times 0.47 "$ & $\begin{array}{c}316 \times 228 \\
(72,048) \\
\end{array}$ \\
\hline & $\begin{array}{c}\text { FTIR } \\
\text { (sweep) }\end{array}$ & $\begin{array}{l}\text { Kinetic Sciences } \\
\text { www.kinetic.bc.ca }\end{array}$ & K-1000 & $\begin{array}{l}\text { up to } \\
1000\end{array}$ & $0.002 " \times 0.6 "$ & $\begin{array}{c}2 \times 900 \\
(\mathrm{H} \times 900)\end{array}$ \\
\hline & FTIR & $\begin{array}{c}\text { Secugen } \\
\text { www.secugen.com } \\
\end{array}$ & Hamster & 500 & $0.64 " \times 0.54 "$ & $\begin{array}{c}320 \times 268 \\
(85,760) \\
\end{array}$ \\
\hline & Sheet prism & $\begin{array}{c}\text { Identix } \\
\text { www.identix.com }\end{array}$ & DFR 200 & 380 & $0.67 " \times 0.67 "$ & $\begin{array}{c}256 \times 256 \\
(65,535)\end{array}$ \\
\hline & Fiber optic & $\begin{array}{c}\text { Delsy } \\
\text { www.delsy.com } \\
\end{array}$ & $\begin{array}{l}\text { CMOS } \\
\text { module }\end{array}$ & 508 & $0.71 " \times 0.47 "$ & $\begin{array}{r}360 \times 240 \\
(86,400) \\
\end{array}$ \\
\hline & $\begin{array}{c}\text { Electro- } \\
\text { optical }\end{array}$ & $\begin{array}{c}\text { Ethentica } \\
\text { www.ethentica.com }\end{array}$ & $\begin{array}{l}\text { TactilSense } \\
\text { T-FPM } \\
\end{array}$ & 403 & $0.76 " \times 0.56 "$ & $\begin{array}{r}306 \times 226 \\
(69,156) \\
\end{array}$ \\
\hline \multirow{7}{*}{ 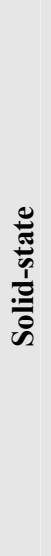 } & $\begin{array}{l}\text { Capacitive } \\
\text { (sweep) }\end{array}$ & $\begin{array}{c}\text { Fujitsu } \\
\text { www.fme.fujitsu.com }\end{array}$ & MBF300 & 500 & $0.06 " \times 0.51 "$ & $\begin{array}{l}32 \times 256 \\
(\mathrm{H} \times 256) \\
\end{array}$ \\
\hline & Capacitive & $\begin{array}{c}\text { Infineon } \\
\text { www.infineon.com } \\
\end{array}$ & FingerTip & 513 & $0.56 " \times 0.44 "$ & $\begin{array}{r}288 \times 224 \\
(64,512) \\
\end{array}$ \\
\hline & Capacitive & $\begin{array}{c}\text { ST-Microelectronics } \\
\text { us.st.com }\end{array}$ & $\begin{array}{l}\text { TouchChip } \\
\text { TCS1AD }\end{array}$ & 508 & $0.71 " \times 0.50 "$ & $\begin{array}{c}360 \times 256 \\
(92,160)\end{array}$ \\
\hline & Capacitive & $\begin{array}{c}\text { Veridicom } \\
\text { www.veridicom.com }\end{array}$ & FPS110 & 500 & $0.60 " \times 0.60 "$ & $\begin{array}{r}300 \times 300 \\
(90,000) \\
\end{array}$ \\
\hline & $\begin{array}{l}\text { Thermal } \\
\text { (sweep) }\end{array}$ & $\begin{array}{c}\text { Atmel } \\
\text { www.atmel.com }\end{array}$ & $\begin{array}{c}\text { FingerChip } \\
\text { AT77C101B }\end{array}$ & 500 & $0.02 " \times 0.55 "$ & $\begin{array}{c}8 \times 280 \\
(\mathrm{H} \times 280)\end{array}$ \\
\hline & Electric field & $\begin{array}{c}\text { Authentec } \\
\text { www.authentec.com }\end{array}$ & AES4000 & 250 & $0.38 " \times 0.38 "$ & $\begin{array}{l}96 \times 96 \\
(9,216) \\
\end{array}$ \\
\hline & Piezoelectric & $\begin{array}{c}\text { BMF } \\
\text { www.bm-f.com }\end{array}$ & BLP-100 & 406 & $0.92 " \times " 0.63$ & $\begin{array}{c}384 \times 256 \\
(98,304)\end{array}$ \\
\hline
\end{tabular}

When analyzed at the global level, the fingerprint pattern exhibits one or more regions where the ridge lines assume distinctive shapes (characterized by high curvature, frequent termination, etc.). These regions (called singularities or singular regions) may be classified into three typologies: loop, delta, and whorl (see Figure 5.b). Singular regions belonging to loop, delta, and whorl types are typically characterized by $\cap, \Delta$, and $\mathrm{O}$ shapes, respectively. Several fingerprint matching algorithms prealign fingerprint images according to a landmark or a center point, called the core. The core point corresponds to the center of the north most loop type singularity. For fingerprints that do not contain loop or whorl singularities (e.g., those belonging to the Arch class in Figure 6), it is difficult to define the core. In these cases, the core is usually associated with the point of maximum ridge line curvature. Unfortunately, due to the high variability of fingerprint patterns, it is difficult to reliably locate a registration (core) point in all the fingerprint images. 
a)

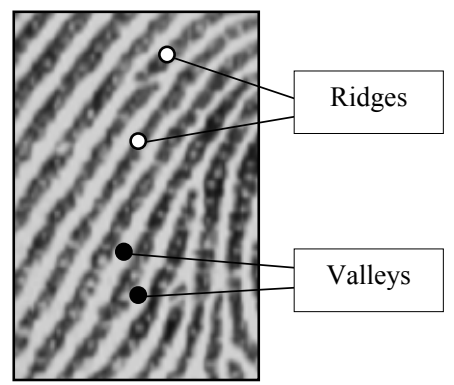

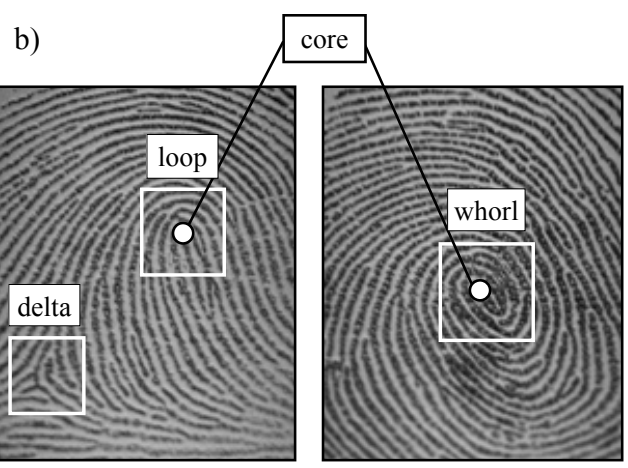

Fig. 5. a) Ridges and valleys on a fingerprint image; b) singular regions (white boxes) and core points (small circles) in fingerprint images.

Singular regions are commonly used for fingerprint classification [21] (see Figure 6), that is, assigning a fingerprint to a class among a set of distinct classes, with the aim of simplifying search and retrieval.

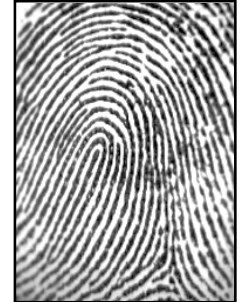

Left loop

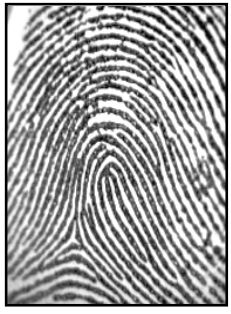

Right loop

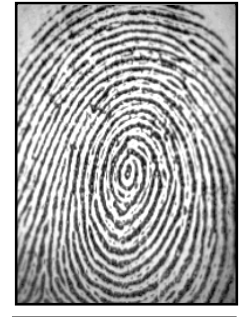

Whorl

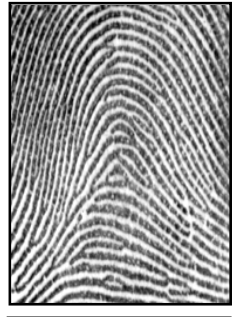

Arch

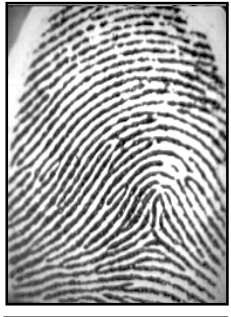

Tented Arch

Fig. 6. One fingerprint from each of the five major classes.

At the local level, other important features, called minutiae can be found in the fingerprint patterns. Minutia refers to various ways that the ridges can be discontinuous. For example, a ridge can suddenly come to an end (termination), or can divide into two ridges (bifurcation). Although several types of minutiae can be considered, usually only a coarse classification is adopted to deal with the practical difficulty in automatically discerning the different types with high accuracy. The FBI minutiaecoordinate model [25] considers only terminations and bifurcations: each minutia is denoted by its class, the $x$ - and $y$-coordinates and the angle between the tangent to the ridge line at the minutia position and the horizontal axis (Figures 7). 

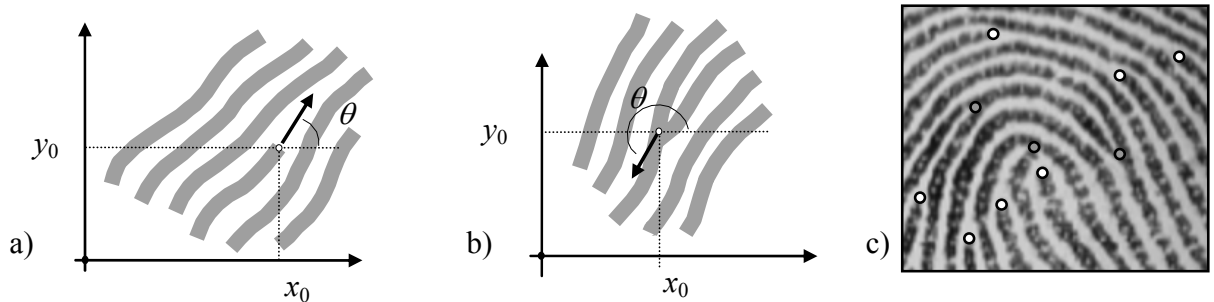

Fig. 7. a) A termination minutia: $\left[x_{0}, y_{0}\right]$ are the minutia coordinates; $\theta$ is the angle that the minutia tangent forms with the horizontal axis; b) a bifurcation minutia: $\theta$ is now defined by means of the termination minutia corresponding to the original bifurcation that exists in the negative image; c) termination (white) and bifurcation (gray) minutiae in a sample fingerprint.

Although some fingerprint matching techniques directly compare images through correlation-based methods, the gray-scale image intensities are known to be an unstable representation. Most of the fingerprint recognition and classification algorithms require a feature extraction stage for identifying salient features. The features extracted from fingerprint images often have a direct physical counterpart (e.g., singularities or minutiae), but sometimes they are not directly related to any physical traits (e.g., local orientation image or filter responses). Features may be used either for matching or their computation may serve as an intermediate step for the derivation of other features. For example, some preprocessing and enhancement steps are often performed to simplify the task of minutiae extraction. Figure 9 provides a graphical representation of the main feature extraction steps and their interrelations.

\subsection{Local Ridge Orientation and Frequency}

The local ridge orientation at $[x, y]$ is the angle $\theta_{x y}$ that the fingerprint ridges, crossing through an arbitrary small neighborhood centered at $[x, y]$, form with the horizontal axis (Figure 8).

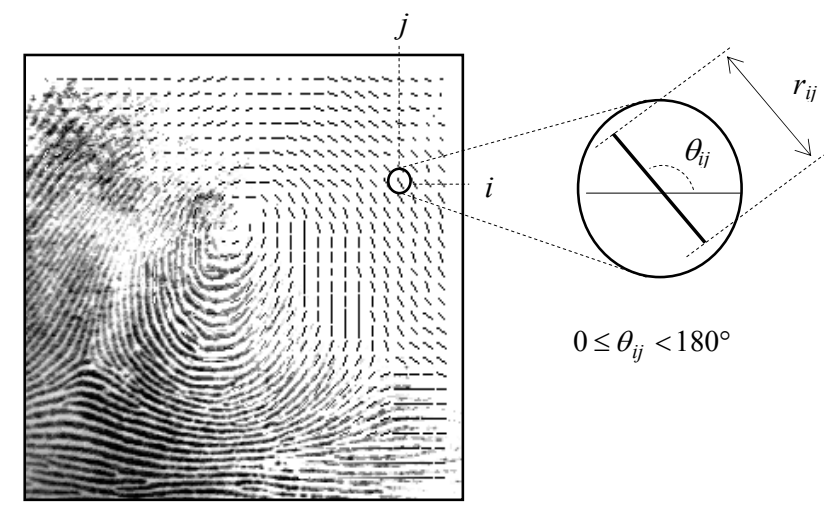

Fig. 8. A fingerprint image faded into the corresponding orientation image computed over a square-meshed grid. Each element denotes the local orientation of the fingerprint ridges; the element length is proportional to its reliability. 


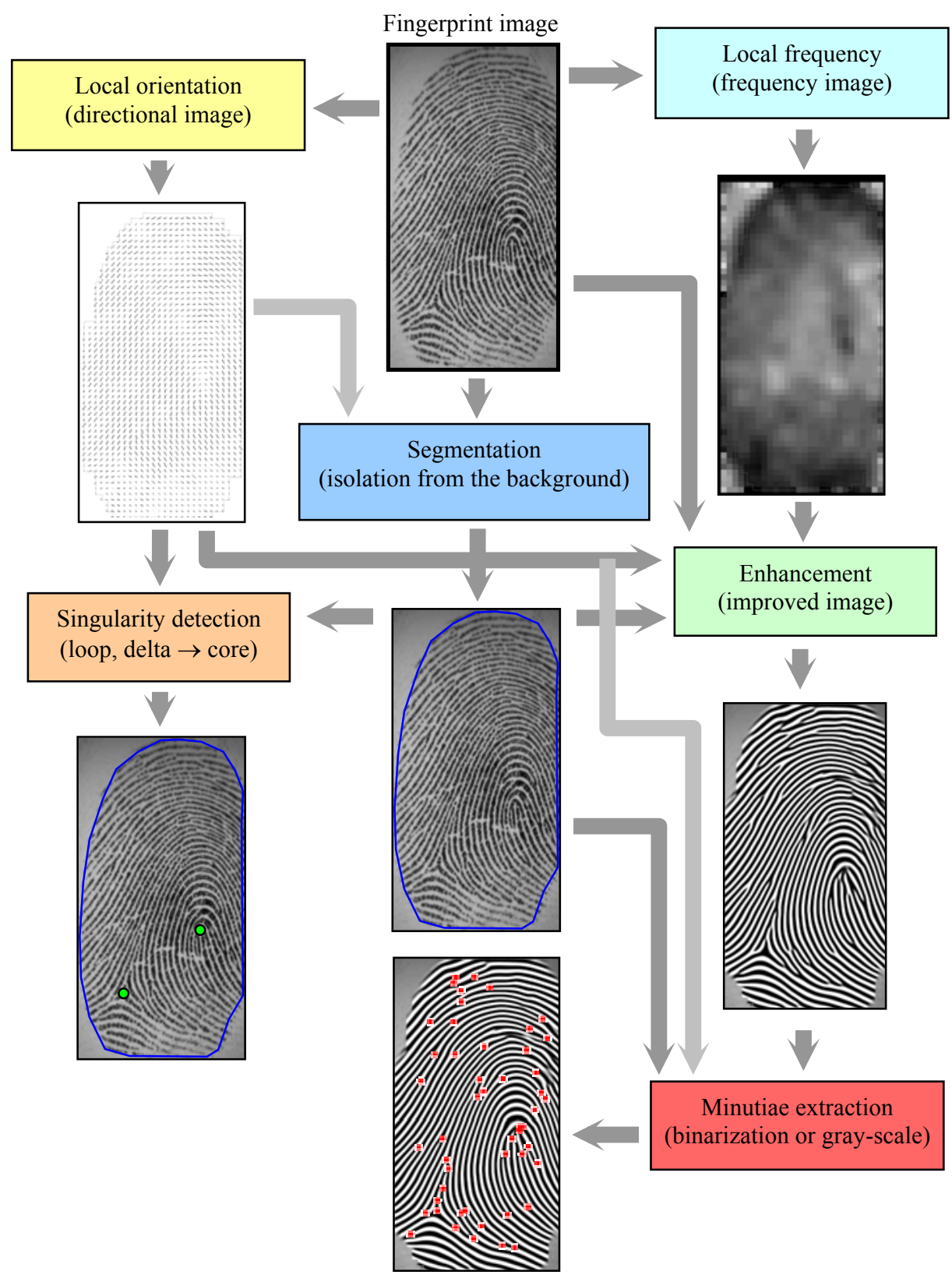

Fig. 9. Graphical representation of fingerprint feature extraction steps and their interrelations. Computation of ridge local orientation and frequency are usually performed at the very beginning since they are useful for most of the other processing steps such as enhancement, singularity detection, segmentation and minutiae extraction. 
The simplest and most natural approach for extracting local ridge orientation is based on computation of gradient phase angles. This method, although simple and efficient, has some drawbacks. First, using the classical convolution masks to determine $\nabla_{x}$ and $\nabla_{y}$ components of the gradient, and computing $\theta_{i j}$ as the arctangent of the $\nabla_{y} / \nabla_{x}$ ratio, presents problems due to the non-linearity and discontinuity around $90^{\circ}$. Second, a single orientation estimate reflects the ridge-valley orientation at too fine a scale and is generally very sensitive to the noise in the fingerprint image.

Robust computation, based on local averaging of gradient estimates, have been proposed by Kass and Witkin [13], Donahue and Rokhlin [7], Ratha, Chen, and Jain [22], and Bazen and Gerez [3].

The local ridge frequency (or density) $f_{x y}$ at point $[x, y]$ is the inverse of the number of ridges per unit length along a hypothetical segment centered at $[x, y]$ and orthogonal to the local ridge orientation $\theta_{x y}$. The local ridge frequency varies across different fingers, and may also noticeably vary across different regions in the same fingerprint (see Figure 10).
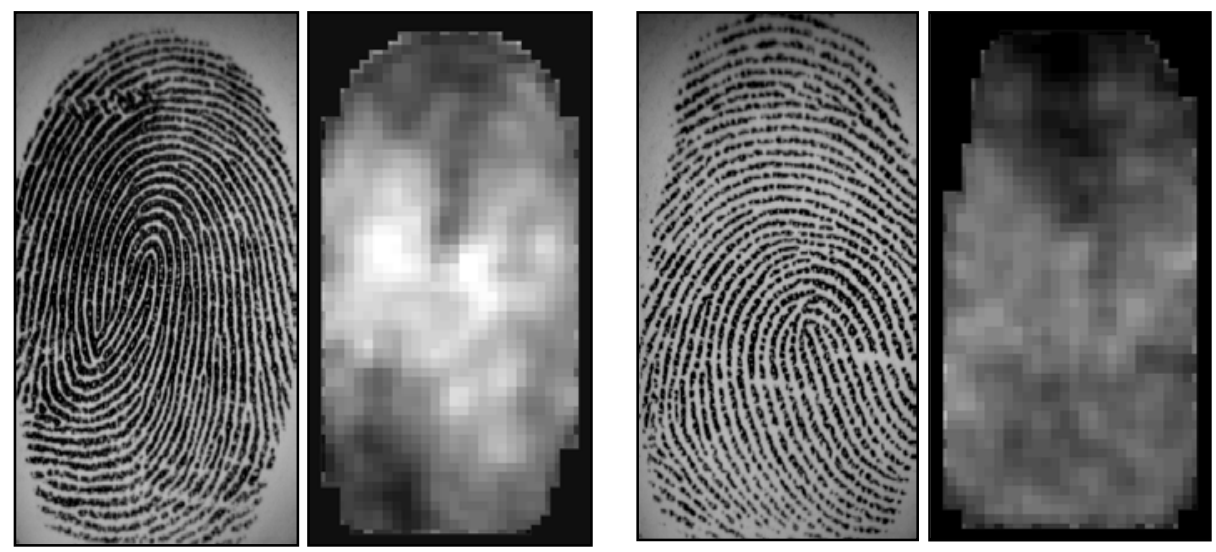

Fig. 10. Two fingerprint images and the corresponding frequency image computed with the method proposed by Maio and Maltoni [20]. Light blocks denote higher frequencies. It is quite evident that significant changes may characterize different fingerprint regions and different average frequencies may result from different fingers.

Hong, Wan, and Jain [10] estimate local ridge frequency by counting the average number of pixels between two consecutive peaks of gray-levels along the direction normal to the local ridge orientation. In the method proposed by Maio and Maltoni [20], the ridge pattern is locally modeled as a sinusoidal-shaped surface, and the variation theorem is exploited to estimate the unknown frequency. Kovacs-Vajna, Rovatti, and Frazzoni [15] proposed a two-step procedure: first, the average ridge distance is estimated in the Fourier domain for each $64 \times 64$ sub-block of the image that is of sufficient quality and then this information is propagated, according to a diffusion equation, to the remaining regions. 


\subsection{Segmentation}

Separating the fingerprint area from the background is useful to avoid extraction of features in noisy areas of the fingerprint and background. Because fingerprint images are striated patterns, using a global or local thresholding technique [9] does not allow the fingerprint area to be effectively isolated. In fact, what really discriminates foreground and background is not the average image intensities but the presence of a striped and oriented pattern in the foreground and of an isotropic pattern (i.e., which does not have a dominant orientation) in the background. If the image background were always uniform and lighter than the fingerprint area, a simple approach based on local intensity could be effective for discriminating foreground and background; in practice, the presence of noise (such as that produced by dust and grease on the surface of live-scan fingerprint scanners) requires more robust segmentation techniques [22][19][2].

\subsection{Singularity Detection}

Most of the approaches proposed in the literature for singularity detection operate on the fingerprint orientation image. The best-known method is based on Poincaré index (Kawagoe and Tojo [14]).

Let $C$ be a closed path defined as an ordered sequence of some elements of the fingerprint orientation image ${ }^{2}$ such that $[i, j]$ is an internal point (see Figure 11), then the Poincaré index $P_{\mathbf{G}, C}(i, j)$ at $[i, j]$ is computed by algebraically summing the orientation differences between adjacent elements of $C$. Summing orientation differences requires a direction (among the two possible) to be associated at each orientation. A solution to this problem is to randomly select the direction of the first element and assign the direction closest to that of the previous element to each successive element.

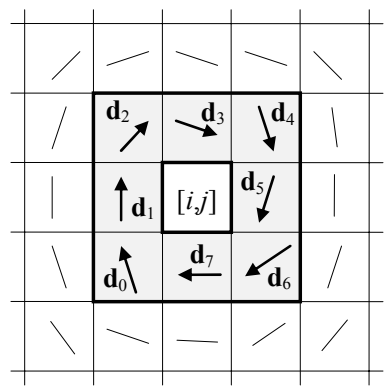

$P_{\mathbf{G}, C}(i, j)=360^{\circ}$

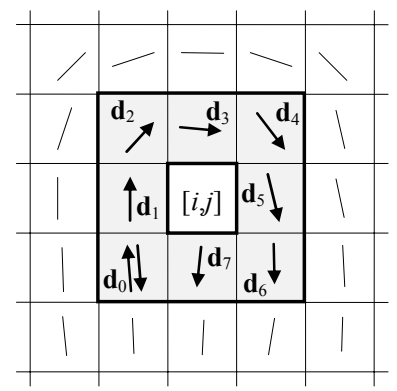

$P_{\mathbf{G}, C}(i, j)=180^{\circ}$

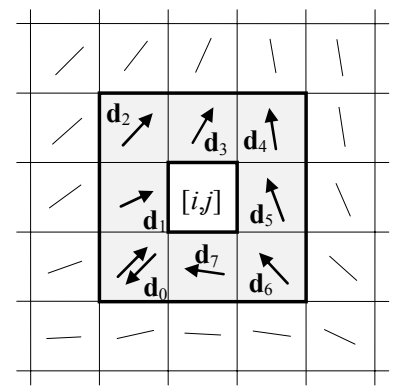

$P_{\mathbf{G}, C}(i, j)=-180^{\circ}$

Fig. 11. Examples of computation of the Poincare index in the 8-neighborhood of points belonging (from the left to the right) to a whorl, loop, and delta singularity, respectively.

${ }^{2}$ The fingerprint orientation image is a matrix whose elements encode the local orientation of the fingerprint ridges. 
It is well known and can be easily shown that, on closed curves, the Poincaré index assumes only one of the discrete values: $0^{\circ}, \pm 180^{\circ}$, and $\pm 360^{\circ}$. In the case of fingerprint singularities:

$$
P_{\mathbf{G}, C}(i, j)=\left\{\begin{aligned}
0^{\circ} & \text { if }[i, j] \text { does not belong to any singular region } \\
360^{\circ} & \text { if }[i, j] \text { belongs to a whorl type singular region } \\
180^{\circ} & \text { if }[i, j] \text { belongs to a loop type singular region } \\
-180^{\circ} & \text { if }[i, j] \text { belongs to a delta type singular region. }
\end{aligned}\right.
$$

An example of singularities detected by the above method is shown in Figure 12.
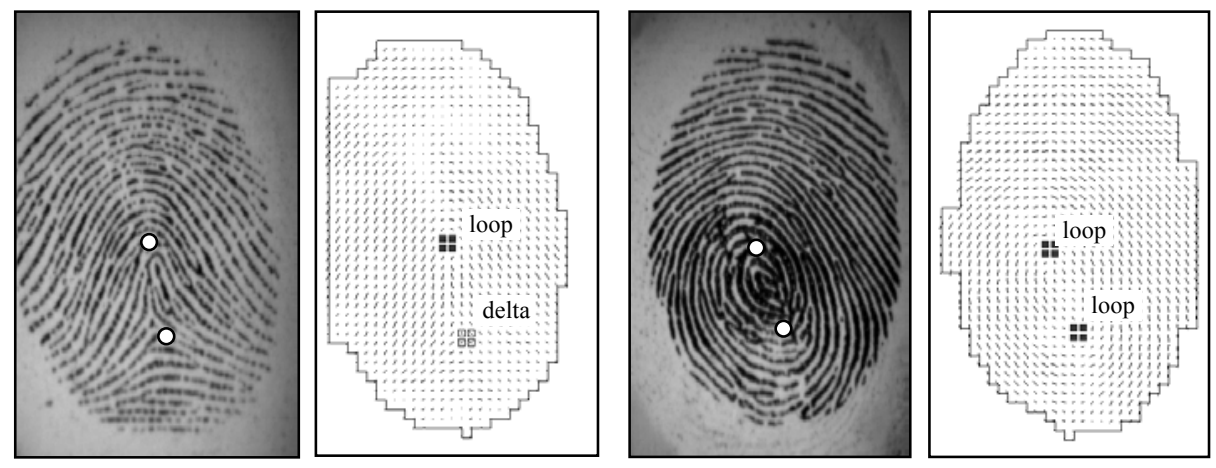

Fig. 12. Singularity detection by using the Poincare index method. The elements whose Poincaré index is $180^{\circ}$ (loop) or $-180^{\circ}$ (delta) are enclosed by small boxes.

A number of alternative approaches have been proposed for singularity detection; they can be coarsely classified in: 1) methods based on local characteristics of the orientation image, 2) partitioning-based methods, 3) core detection and fingerprint registration approaches. For further details refer to [21].

\subsection{Enhancement and Binarization}

The performance of minutiae extraction algorithms and other fingerprint recognition techniques relies heavily on the quality of the input fingerprint images. In an ideal fingerprint image, ridges and valleys alternate and flow in a locally constant direction. In such situations, the ridges can be easily detected and minutiae can be precisely located in the image. However, in practice, due to skin conditions (e.g., wet or dry, cuts, and bruises), sensor noise, incorrect finger pressure, and inherently low-quality fingers (e.g., elderly people, manual workers), a significant percentage of fingerprint images (approximately 10\%) is of poor quality like those in Figures 2.b, c and d.

The goal of an enhancement algorithm is to improve the clarity of the ridge structures in the recoverable regions and mark the unrecoverable regions as too noisy for further processing. Usually, the input of the enhancement algorithm is a gray-scale image. The output may either be a gray-scale or a binary image, depending on the algorithm. 
General-purpose image enhancement techniques do not produce satisfying and definitive results for fingerprint image enhancement. The most widely used technique for fingerprint image enhancement is based on contextual filters. In conventional image filtering, only a single filter is used for convolution throughout the image. In contextual filtering, the filter characteristics change according to the local context. Usually, a set of filters is pre-computed and one of them is selected for each image region. In fingerprint enhancement, the context is often defined by the local ridge orientation and local ridge frequency. In fact, the sinusoidal-shaped wave of ridges and valleys is mainly defined by a local orientation and frequency that varies slowly across the fingerprint area. An appropriate filter that is tuned to the local ridge frequency and orientation can efficiently remove the undesired noise and preserve the true ridge and valley structure.

Hong, Wan, and Jain [10] proposed an effective method based on Gabor filters. Gabor filters have both frequency-selective and orientation-selective properties and have optimal joint resolution in both spatial and frequency domains. A graphical representation of a bank of 24 filters and an example of their applications is shown in Figure 13. Further information on the huge number of existing fingerprint enhancement and binarization techniques can be found in [21].

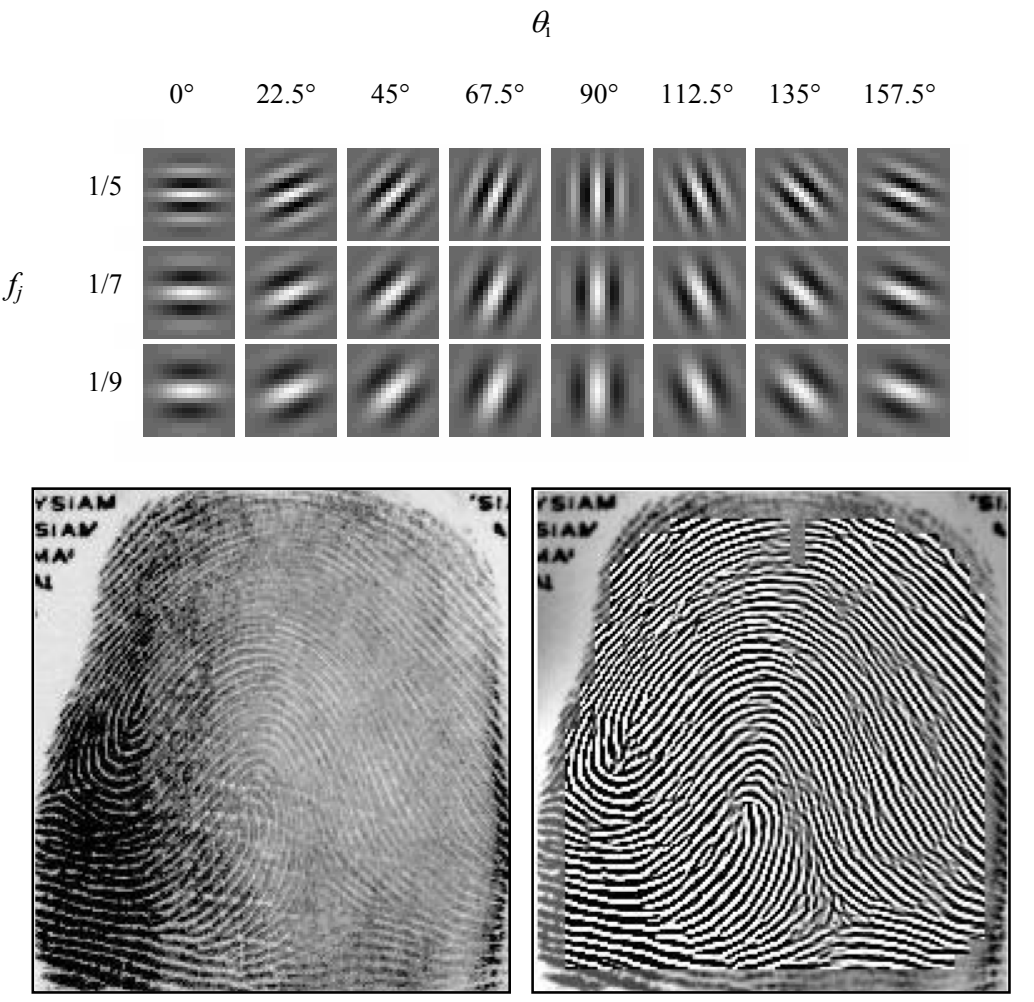

Fig. 13. A graphical representation of a bank of 24 Gabor filters and their application to the enhancement of a noisy image. 


\subsection{Minutiae Extraction}

Although rather different from one another, most of the proposed methods require the fingerprint gray-scale image to be converted into a binary image. Some binarization processes greatly benefit from an a priori enhancement; on the other hand, some enhancement algorithms directly produce a binary output, and therefore the distinction between enhancement and binarization is often faded. The binary images obtained by the binarization process are usually submitted to a thinning stage [16] which allows for the ridge line thickness to be reduced to one pixel. Finally, a simple image scan allows the detection of pixels that correspond to minutiae through the pixel-wise computation of crossing number ${ }^{3}$ (see Figure 14).

a)

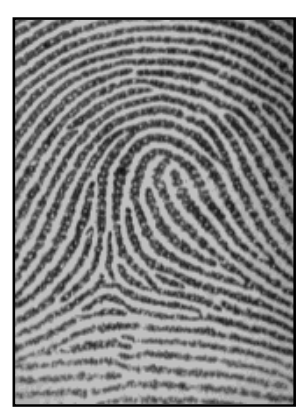

b)

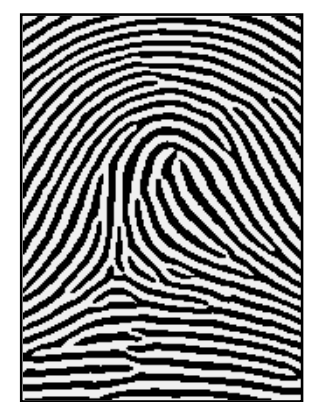

c)

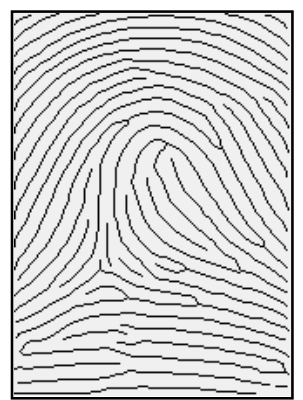

d)

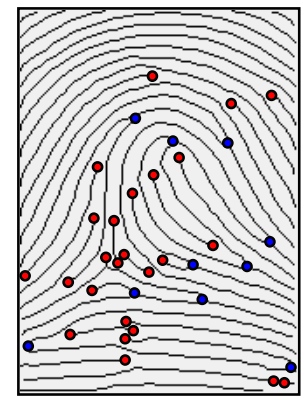

Fig. 14. a) A fingerprint gray-scale image; b) the image obtained after enhancement and binarization; c) the image obtained after thinning; d) termination and bifurcation minutiae detected through the pixel-wise computation of the crossing number.

Some authors have proposed minutiae extraction approaches that work directly on the gray-scale images without binarization and thinning. This choice is motivated by these considerations:

- a significant amount of information may be lost during the binarization process;

- binarization and thinning are time consuming; thinning may introduce a large number of spurious minutiae;

- in the absence of an a priori enhancement step, most of the binarization techniques do not provide satisfactory results when applied to low-quality images.

Maio and Maltoni [19] proposed a direct gray-scale minutiae extraction technique, whose basic idea is to track the ridge lines in the gray-scale image, by "sailing" according to the local orientation of the ridge pattern. The ridge line extraction algorithm attempts to locate, at each step, a local maximum relative to a section orthogonal to the ridge direction. By connecting the consecutive maxima, a polygonal ap-

${ }^{3}$ The crossing number of a pixel in a binary image is defined as half the sum of the differences between pairs of adjacent pixels in the 8-neighborhood [21]; its value is 1 for a termination minutia, 2 for an intermediate ridge pixel, and $\geq 3$ for a bifurcation or a more complex minutia. 
proximation of the ridge line can be obtained. See Figure 15 for an example of direct gray-scale minutiae extraction.
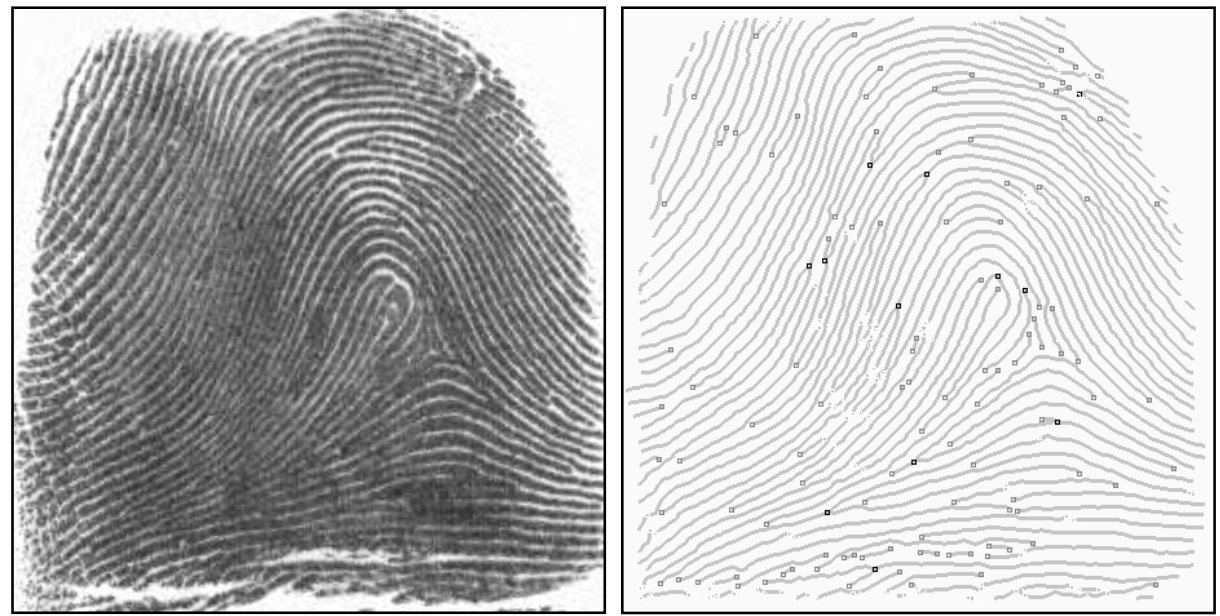

Fig. 15. Minutiae detection on a sample fingerprint by using the Maio and Maltoni [19] method.

A post-processing stage (called minutiae filtering) is often useful in removing the spurious minutiae detected in highly corrupted regions or introduced by previous processing steps (e.g., thinning). Two main post-processing types have been proposed: structural post-processing, and minutiae filtering in the gray-scale domain [21].

\section{Matching}

Matching high quality fingerprints with small intra-class variations is not difficult and every reasonable algorithm can do it. The real challenge is matching samples (sometimes very poor quality) affected by:

- High displacement and/or rotation: finger displacement and rotation often cause part of the fingerprint area to fall outside the sensor's "field of view," resulting in a smaller overlap between the template and the input fingerprints. This problem is particularly serious for small-area sensors. A finger displacement of just $2 \mathrm{~mm}$ (imperceptible to the user) results in a translation of about 40 pixels in a fingerprint image scanned at $500 \mathrm{dpi}$.

- Non-linear distortion: the act of sensing maps the three-dimensional shape of a finger onto the two-dimensional surface of the sensor. This results in a non-linear distortion in successive acquisitions of the same finger due to skin plasticity.

- Different pressure and skin condition: the ridge structure of a finger would be accurately captured if ridges of the part of the finger being imaged were in uniform contact with the sensor surface. However, finger pressure, dryness of the 
skin, skin disease, sweat, dirt, grease, and humidity in the air all confound the situation, resulting in a non-uniform contact.

- Feature extraction errors: the feature extraction algorithms are imperfect and often introduce measurement errors. For example in low-quality fingerprint images, the minutiae extraction process may introduce a large number of spurious minutiae and may not be able to detect all the true minutiae.

The pairs of images in Figure 16.a visually show the high variability (large intraclass variations) that can characterize two different impressions of the same finger. On the other hand, as evident from Figure 16.b, fingerprint images from different fingers may sometimes appear quite similar (small inter-class variations), especially in terms of global structure (position of the singularities, local ridge orientation, etc.).

a)

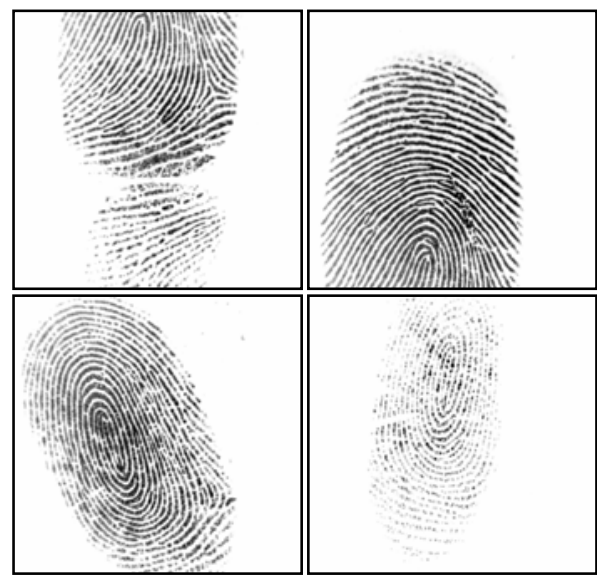

b)

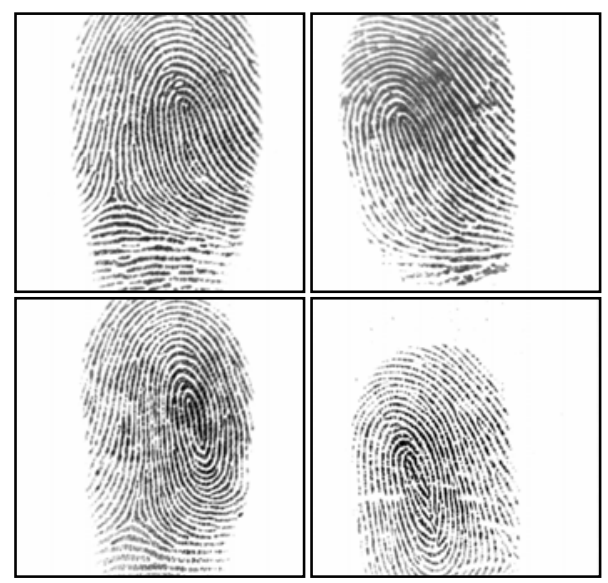

Fig. 16. a) each row shows a pair of impressions of the same finger, taken from the FVC2002 DB1, which were falsely non-matched by most of the algorithms submitted to FVC2002 [18]. The main cause of difficulty is a very small overlap in the first row, and very different skin conditions in the second row; b) each row shows a pair of impressions of different fingers, taken from the FVC2002 databases which were falsely matched by some of the algorithms submitted to FVC2002.

The large number of existing approaches to fingerprint matching can be coarsely classified into three families.

- Correlation-based matching: two fingerprint images are superimposed and the correlation between corresponding pixels is computed for different alignments (e.g., various displacements and rotations).

- Minutiae-based matching: minutiae are extracted from the two fingerprints and stored as sets of points in the two-dimensional plane. Minutiae-based matching essentially consists of finding the alignment between the template and the input minutiae sets that results in the maximum number of minutiae pairings.

- Ridge feature-based matching: the approaches belonging to this family compare fingerprints in term of features extracted from the ridge pattern. 
In the rest of this section the representation of the fingerprint acquired during enrollment is denoted to as the template (T) and the representation of the fingerprint to be matched as the input (I). In case no feature extraction is performed, the fingerprint representation coincides with the grayscale fingerprint image itself.

\subsection{Correlation-Based Techniques}

Let $\mathbf{I}^{(\Delta x, \Delta y, \theta)}$ represent a rotation of the input image $\mathbf{I}$ by an angle $\theta$ around the origin (usually the image center) and shifted by $\Delta x, \Delta y$ pixels in directions $x$ and $y$, respectively; then the similarity between the two fingerprint images $\mathbf{T}$ and $\mathbf{I}$ can be measured as

$$
S(\mathbf{T}, \mathbf{I})=\max _{\Delta x, \Delta y, \theta} C C\left(\mathbf{T}, \mathbf{I}^{(\Delta x, \Delta y, \theta)}\right) .
$$

where $C C(\mathbf{T}, \mathbf{I})=\mathbf{T}^{\mathrm{T}} \mathbf{I}$ is the cross-correlation between $\mathbf{T}$ and $\mathbf{I}$. The cross-correlation [9] is well known measure of image similarity and the maximization in (1) allows to find the optimal registration.

Anyway, the direct application of Equation (1) rarely leads to acceptable results mainly due to the following problems.

- Non-linear distortion makes impressions of the same finger significantly different in terms of global structure; in particular, the elastic distortion does not significantly alter the fingerprint pattern locally, but since the effects of distortion get integrated in image space, two global fingerprint patterns cannot be reliably correlated. The use of local or block-wise correlation techniques can help to deal with this problem [4].

- Skin condition and finger pressure cause image brightness, contrast, and ridge thickness to vary significantly across different impressions. The use of more sophisticated correlation measures may compensate for contrast and brightness variations and applying a proper combination of enhancement, binarization, and thinning steps (performed on both $\mathbf{T}$ and $\mathbf{I}$ ) may limit the ridge thickness problem.

- A direct application of Equation (1) is computationally very expensive. For example, consider two $400 \times 400$ pixel images: if $\Delta x, \Delta y$ were both sampled with a onepixel step in the range $[-200,200]$, and $\theta$ with step $1^{\circ}$ in the range $\left[-30^{\circ}, 30^{\circ}\right]$ it would be necessary to compute $401 \times 401 \times 61$ cross-correlations, resulting in about 1569 billion multiplications and summations (i.e., more than one hour on a 500 MIPS computer). Local correlation and correlation in the Fourier domain can improve efficiency.

\subsection{Minutiae-Based Methods}

This is the most popular and widely used technique, being the basis of the fingerprint comparison made by fingerprint examiners. Minutiae are extracted from the two fingerprints and stored as sets of points in the two-dimensional plane. Most common 
minutiae matching algorithms consider each minutia as a triplet $\mathbf{m}=\{x, y, \theta\}$ that indicates the $x, y$ minutia location coordinates and the minutia angle $\theta$ :

$$
\begin{array}{ll}
\mathbf{T}=\left\{\mathbf{m}_{1}, \mathbf{m}_{2}, \ldots, \mathbf{m}_{m}\right\}, & \mathbf{m}_{i}=\left\{x_{i}, y_{i}, \theta_{i}\right\}, \quad i=1 . . m \\
\mathbf{I}=\left\{\mathbf{m}_{1}^{\prime}, \mathbf{m}_{2}^{\prime}, \ldots, \mathbf{m}_{n}^{\prime}\right\}, & \mathbf{m}_{j}^{\prime}=\left\{x_{j}^{\prime}, y_{j}^{\prime}, \theta_{j}^{\prime}\right\}, \quad j=1 . . n,
\end{array}
$$

where $m$ and $n$ denote the number of minutiae in $\mathbf{T}$ and $\mathbf{I}$, respectively.

A minutia $\mathbf{m}_{j}^{\prime}$ in $\mathbf{I}$ and a minutia $\mathbf{m}_{i}$ in $\mathbf{T}$ are considered "matching," if the spatial distance $(s d)$ between them is smaller than a given tolerance $r_{0}$ and the direction difference $(d d)$ between them is smaller than an angular tolerance $\theta_{0}$ :

$$
\begin{aligned}
& s d\left(\mathbf{m}_{j}^{\prime}, \mathbf{m}_{i}\right)=\sqrt{\left(x_{j}^{\prime}-x_{i}\right)^{2}+\left(y_{j}^{\prime}-y_{i}\right)^{2}} \leq r_{0,} \quad \text { and } \\
& d d\left(\mathbf{m}_{j}^{\prime}, \mathbf{m}_{i}\right)=\min \left(\left|\theta_{j}^{\prime}-\theta_{i}\right|, 360^{\circ}-\left|\theta_{j}^{\prime}-\theta_{i}\right|\right) \leq \theta_{0} .
\end{aligned}
$$

The tolerance boxes (or hyper-spheres) defined by $r_{0}$ and $\theta_{0}$ are necessary to compensate for the unavoidable errors made by feature extraction algorithms and to account for the small plastic distortions that cause the minutiae positions to change.

Aligning the two fingerprints is a mandatory step in order to maximize the number of matching minutiae. Correctly aligning two fingerprints certainly requires displacement (in $x$ and $y$ ) and rotation $(\theta)$ to be recovered, and likely involves other geometrical transformations like scale and specific distortion-tolerant geometrical transformations. Let $\operatorname{map}\left(\right.$.) be the function that maps a minutia $\mathbf{m}_{j}^{\prime}$ (from I) into $\mathbf{m}_{j}^{\prime \prime}$ according to a given geometrical transformation; for example, by considering a displacement of $[\Delta x, \Delta y]$ and a counterclockwise rotation $\theta$ around the origin ${ }^{4}$ :

$$
\begin{aligned}
& \operatorname{map}_{\Delta x, \Delta y, \theta}\left(\mathbf{m}_{j}^{\prime}=\left\{x_{j}^{\prime}, y_{j}^{\prime}, \theta_{j}^{\prime}\right\}\right)=\mathbf{m}_{j}^{\prime \prime}=\left\{x_{j}^{\prime \prime}, y_{j}^{\prime \prime}, \theta_{j}^{\prime}+\theta\right\}, \quad \text { where } \\
& {\left[\begin{array}{c}
x_{j}^{\prime \prime} \\
y_{j}^{\prime \prime}
\end{array}\right]=\left[\begin{array}{cc}
\cos \theta & -\sin \theta \\
\sin \theta & \cos \theta
\end{array}\right]\left[\begin{array}{l}
x_{j}^{\prime} \\
y_{j}^{\prime}
\end{array}\right]+\left[\begin{array}{c}
\Delta x \\
\Delta y
\end{array}\right] .}
\end{aligned}
$$

Let $m m($.$) be an indicator function that returns 1$ in the case where the minutiae $\mathbf{m}_{j}^{\prime \prime}$ and $\mathbf{m}_{i}$ match according to Equations (2) and (3):

$$
m m\left(\mathbf{m}_{j}^{\prime \prime}, \mathbf{m}_{i}\right)= \begin{cases}1 & s d\left(\mathbf{m}_{j}^{\prime \prime}, \mathbf{m}_{i}\right) \leq r_{0} \quad \text { and } \quad d d\left(\mathbf{m}_{j}^{\prime \prime}, \mathbf{m}_{i}\right) \leq \theta_{0} \\ 0 & \text { otherwise. }\end{cases}
$$

Then, the matching problem can be formulated as

$$
\underset{\Delta x, \Delta y, \theta, P}{\operatorname{maximize}} \sum_{i=1}^{m} m m\left(\operatorname{map}_{\Delta x, \Delta y, \theta}\left(\mathbf{m}_{P(i)}^{\prime}\right), \mathbf{m}_{i}\right)
$$

where $P(i)$ is an unknown function that determines the pairing between $\mathbf{I}$ and $\mathbf{T}$ minutiae; in particular, each minutia has either exactly one mate in the other fingerprint or has no mate at all:

4 The origin is usually selected as the minutiae centroid (i.e., the average point). 
1. $P(i)=j$ indicates that the mate of the $\mathbf{m}_{i}$ in $\mathbf{T}$ is the minutia $\mathbf{m}_{j}^{\prime}$ in $\mathbf{I}$;

2. $P(i)=$ null indicates that minutia $\mathbf{m}_{i}$ in $\mathbf{T}$ has no mate in $\mathbf{I}$;

3. a minutia $\mathbf{m}_{j}^{\prime}$ in $\mathbf{I}$, such that $\forall i=1 . . m, P(i) \neq j$ has no mate in $\mathbf{T}$;

4. $\forall i=1 . . m, k=1 . . m, i \neq k \Rightarrow P(i) \neq P(k)$ or $P(i)=P(k)=$ null (this requires that each minutia in $\mathbf{I}$ is associated with a maximum of one minutia in $\mathbf{T}$ ).

Note that, in general, $P(i)=j$ does not necessarily mean that minutiae $\mathbf{m}_{j}^{\prime}$ and $\mathbf{m}_{i}$ match in the sense of Equations (2) and (3) but only that they are the most likely pair under the current transformation.

Solving the minutiae matching problem (expression (4)) is trivial if the correct alignment ( $\Delta x, \Delta y, \theta)$ or the function $P$ (minutiae correspondence) is known. Unfortunately, in practice, neither the alignment parameters nor the correspondence function $P$ are known and, therefore, solving the matching problem is very hard. In the pattern recognition literature the minutiae matching problem has been generally addressed as a point pattern matching problem [21]. Hough transform-based approaches are the most commonly used techniques for global minutiae matching [23] [5]; an example is shown in Figure 17. The Hough transform techniques [1] converts point pattern matching to the problem of detecting peaks in the Hough space of transformation parameters. It discretizes the parameter space $(\Delta x, \Delta y, \theta)$ and accumulates evidence in the discretized space by deriving transformation parameters that relate two sets of points using a substructure of the feature matching technique.

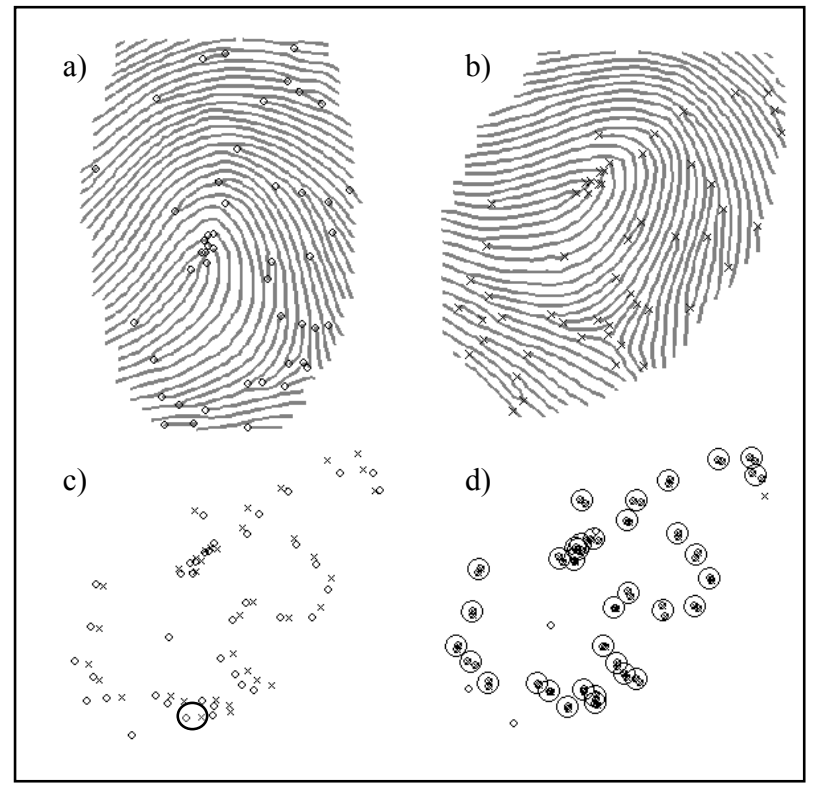

Fig. 17. Minutiae matching by the Chang et al. approach [5]. Figures a) and b) show the minutiae extracted from the template and the input fingerprint, respectively; c) the minutiae are coarsely superimposed and the principal pair is marked with an ellipse; d) each circle denotes a pair of minutiae as mated by the algorithm. 
Equation 4 attempts to solve the minutiae matching problem globally (global $\mathrm{mi}$ nutiae matching). Some authors proposed "local minutiae matching" techniques that consists of comparing two fingerprints according to local minutiae structures (Jiang and Yau [12], Ratha et al. [24]); local structures are characterized by attributes that are invariant with respect to global transformation (e.g., translation, rotation, etc.) and therefore are suitable for matching without any a priori global alignment. Matching fingerprints based only on local minutiae arrangements relaxes global spatial relationships which are highly distinctive and therefore reduce the amount of information available for discriminating fingerprints. Global versus local matching is a tradeoff among simplicity, low computational complexity, and high distortion-tolerance (local matching), and high distinctiveness on the other hand (global matching).

In [12] local structures are formed by a central minutia and its two nearestneighbor minutiae; the feature vector $\mathbf{v}_{i}$ associated with the minutia $\mathbf{m}_{i}$, whose nearest neighbors are minutiae $\mathbf{m}_{j}$ (the closest to $\mathbf{m}_{i}$ ) and $\mathbf{m}_{k}$ (the second closest) is $\mathbf{v}_{i}=\left[d_{i j}\right.$, $\left.d_{i k}, \theta_{i j}, \theta_{i k}, \varphi_{i j}, \varphi_{i k}, n_{i j}, n_{i k}, t_{i}, t_{j}, t_{k}\right]$, where $d_{a b}$ is the distance between minutiae $\mathbf{m}_{a}$ and $\mathbf{m}_{b}, \theta_{a b}$ is the direction difference between the angles $\theta_{a}$ and $\theta_{b}$ of $\mathbf{m}_{a}$ and $\mathbf{m}_{b}, \varphi_{a b}$ is the direction difference between the angle $\theta_{a}$ of $\mathbf{m}_{a}$ and the direction of the edge connecting $\mathbf{m}_{a}$ to $\mathbf{m}_{b}, n_{a b}$ is the ridge count between $\mathbf{m}_{a}$ and $\mathbf{m}_{b}$, and $t_{a}$ is the minutia type of $\mathbf{m}_{a}$ (Figure 18).

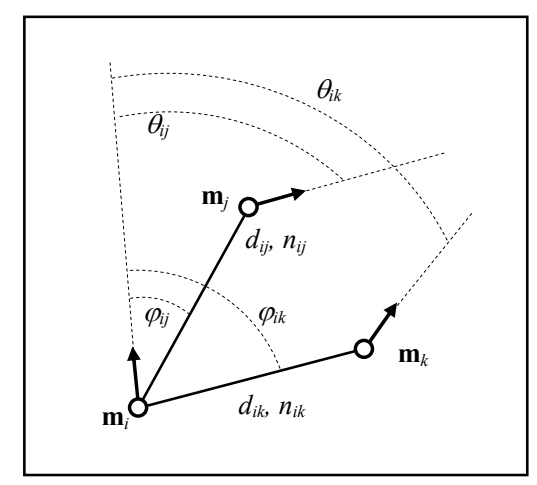

Fig. 18. Features of the local structures used by Jiang and Yau [12].

Local minutiae matching is performed by computing, for each pair of minutiae $\mathbf{m}_{i}$ and $\mathbf{m}_{j}^{\prime}, i=1 . . m, j=1 . . n$, a weighted distance between their vectors $\mathbf{v}_{i}$ and $\mathbf{v}_{j}^{\prime}$. The best matching pair is then selected and used for registering the two fingerprints. In the second stage (consolidation), the feature vectors of the remaining aligned pairs are matched and a final score is computed by taking into account the different contributions (first stage and second stage). 


\subsection{Ridge Feature-Based Techniques}

Three main reasons induce designers of fingerprint recognition techniques to search for other fingerprint distinguishing features, beyond minutiae: 1) reliably extracting minutiae from poor quality fingerprints is very difficult; 2 ) minutiae extraction is time consuming; 3) additional features may be used in conjunction with minutiae (and not as an alternative) to increase system accuracy and robustness. The more commonly used alternative features are:

1. size of the fingerprint and shape of the external fingerprint silhouette;

2. number, type, and position of singularities;

3. spatial relationship and geometrical attributes of the ridge lines;

4. shape features;

5. global and local texture information;

6. sweat pores;

7. fractal features.

Jain et al. [11] proposed a local texture analysis technique where the fingerprint area of interest is tessellated with respect to the core point (see Figure 19).

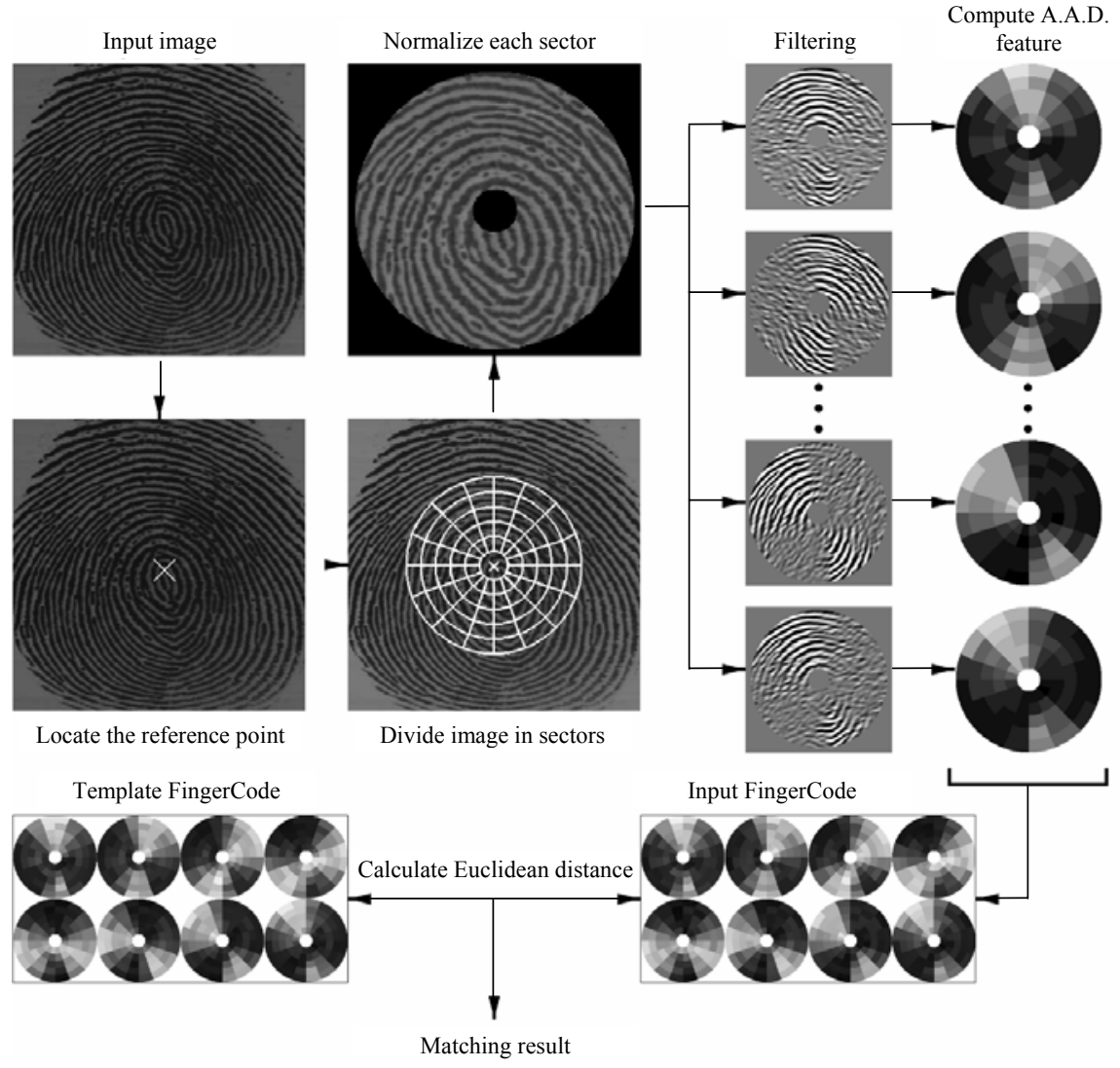

Fig. 19. System diagram of Jain et al.'s FingerCode approach [11]. 
A feature vector is composed of an ordered enumeration of the features extracted from the local information contained in each sector specified by the tessellation. Thus the feature elements capture the local texture information and the ordered enumeration of the tessellation captures the global relationship among the local contributions. The local texture information in each sector is decomposed into separate channels by using a Gabor filterbank. Each fingerprint is represented by a $80 \times 8=640$ fixed-size feature vector, called the FingerCode. The generic element $\mathrm{V}_{i j}$ of the vector $(i=1 . .80$ is the cell index, $j=1 . .8$ is the filter index) denotes the energy revealed by the filter $j$ in cell $i$, and is computed as the average absolute deviation (AAD) from the mean of the responses of the filter $j$ over all the pixels of the cell $i$. Matching two fingerprints is then translated into matching their respective FingerCodes, which is simply performed by computing the Euclidean distance between two FingerCodes.

\section{Conclusions}

Recent developments in fingerprint scanners have focused on reducing both their cost and size. Although lower cost and size are essential to enable a wide deployment of the technology in civilian applications, some of these developments have been made at the expense of fingerprint image quality (e.g., dpi resolution, etc.). It is very likely that while the market will continue to drive down scanner prices, it will also require higher-quality products at the same time. Manufacturers will continue to innovate low-cost small-size scanner designs, but they will also take care that their products deliver high quality-images of large areas of the finger.

Robust extraction of fingerprint feature remains a challenging problem, especially in poor quality fingerprints. Development of fingerprint-specific image processing techniques is necessary in order to solve some of the outstanding problems. For example, explicitly measuring (and restoring or masking) noise such as creases, cuts, dryness, smudginess, and the like will be helpful in reducing feature extraction errors. Algorithms that can extract discriminative non-minutiae-based features in fingerprint images and integrate them with the available features and matching strategies will improve fingerprint matching accuracy. New (perhaps, model-based) methods for computation (or restoration) of the orientation image in very low-quality images is also desirable to reduce feature extraction errors.

Most of the fingerprint matching approaches introduced in the last four decades are minutiae-based, but recently correlation-based techniques are receiving renewed interest. New texture-based methods have been proposed and the integration of approaches relying on different features seems to be the most promising way to significantly improve the accuracy of fingerprint recognition systems.

\section{References}

1. Ballard, D.H.: Generalizing the Hough Transform to Detect Arbitrary Shapes. Pattern Recognition, vol. 3, no. 2 (1981) 110-122. 
2. Bazen, A.M., Gerez, S.H.: Segmentation of Fingerprint Images. Proc. Workshop on Circuits Systems and Signal Processing (ProRISC 2001) (2001) 276-280.

3. Bazen, A.M., Gerez, S.H.: Systematic Methods for the Computation of the Directional Fields and Singular Points of Fingerprints. IEEE Transactions on Pattern Analysis and Machine Intelligence, vol. 24, no. 7 (2002) 905-919.

4. Bazen, A.M., Verwaaijen, G.T.B., Gerez, S.H., Veelenturf, L.P.J., van der Zwaag, B.J.: A Correlation-Based Fingerprint Verification System. Proc. Workshop on Circuits Systems and Signal Processing (ProRISC 2000) (2000) 205-213.

5. Chang, S.H., Cheng, F.H., Hsu, W.H., and Wu, G.Z.: Fast Algorithm for Point PatternMatching: Invariant to Translations, Rotations and Scale Changes. Pattern Recognition, vol. 30, no. 2 (1997) 311-320.

6. Criminal Justice Information Services: Electronic Fingerprint Transmission Specification. Int. Report. CJIS-RS-0010 (V7), (1999), available at: http://www.fbi.gov/hq/cjisd/iafis/ efts70/cover.htm.

7. Donahue, M.L, Rokhlin, S.I.: On the use of Level Curves in Image Analysis. CVGIP: Image Understanding, vol. 57, no. 2 (1993) 185-203.

8. Golfarelli, M., Maio, D., Maltoni, D.: On the Error-Reject Tradeoff in Biometric Verification Systems. IEEE Transactions on Pattern Analysis and Machine Intelligence, vol. 19, no.7, (1997) 786-796.

9. Gonzales, R.C., Woods, R.E.: Digital Image Processing. Addison-Wesley, Reading, MA (1992).

10. Hong, L., Wan, Y., Jain, A.K.: Fingerprint Image Enhancement: Algorithms and Performance Evaluation. IEEE Transactions on Pattern Analysis and Machine Intelligence, vol. 20, no. 8 (1998) 777-789.

11. Jain, A.K., Prabhakar, S., Hong, L., Pankanti, S.: Filterbank-Based Fingerprint Matching. IEEE Transactions on Image Processing, vol. 9 (2000) 846-859.

12. Jiang, X., Yau, W.Y.: Fingerprint Minutiae Matching Based on the Local and Global Structures. Proc. Int. Conf. on Pattern Recognition (15th), vol. 2 (2000) 1042-1045.

13. Kass, M., Witkin, A.: Analyzing Oriented Patterns. Computer Vision, Graphics, and Image Processing, vol. 37, no. 3 (1987) 362-385.

14. Kawagoe, M., Tojo, A.: Fingerprint Pattern Classification. Pattern Recognition, vol. 17, (1984) 295-303.

15. Kovacs-Vajna, Z.M., Rovatti, R., Frazzoni, M.: Fingerprint Ridge Distance Computation Methodologies. Pattern Recognition, vol. 33, no. 1 (2000) 69-80.

16. Lam, L., Lee, S.W., Suen, C.Y.: Thinning Methodologies: A Comprehensive Survey. IEEE Transactions on Pattern Analysis and Machine Intelligence, vol. 14, no. 9 (1992) 869-885.

17. Lee, H.C., Gaensslen, R.E.: Advances in Fingerprint Technology. 2nd edition, Elsevier, New York (2001).

18. Maio, D., Maltoni, D., Cappelli, R., Wayman, J.L., Jain A.K.: FVC2002: Second Fingerprint Verification Competition. Proc. Int. Conf. on Pattern Recognition (16th), vol. 3 (2002) 811-814.

19. Maio, D., Maltoni, D.: Direct Gray-Scale Minutiae Detection in Fingerprints. IEEE Transactions on Pattern Analysis and Machine Intelligence, vol. 19, no. 1 (1997).

20. Maio, D., Maltoni, D.: Ridge-Line Density Estimation in Digital Images. Proc. Int. Conf. on Pattern Recognition (14th) (1998) 534-538.

21. Maltoni, D., Maio, D., Jain, A.K., Prabhakar, S.: Handbook of Fingerprint Recognition, Springer, New York (2003). 
22. Ratha, N.K., Chen, S.Y., Jain, A.K.: Adaptive Flow Orientation-Based Feature Extraction in Fingerprint Images. Pattern Recognition, vol. 28, no. 11 (1995) 1657-1672.

23. Ratha, N.K., Karu, K., Chen, S., Jain, A.K.: A Real-Time Matching System for Large Fingerprint Databases. IEEE Transactions on Pattern Analysis and Machine Intelligence, vol. 18, no. 8 (1996) 799-813.

24. Ratha, N.K., Pandit, V.D., Bolle, R.M., Vaish, V.: Robust Fingerprint Authentication Using Local Structural Similarity. Proc. Workshop on Applications of Computer Vision (2000) 29-34.

25. Wegstein, J.H.: An Automated Fingerprint Identification System. U.S. Government Publication, Washington, DC: U.S. Dept. of Commerce, National Bureau of Standards (1982). 